\title{
The Technique of Angioplasty and Stent Placement in Acute Ischemic Stroke Therapy
}

\author{
Shah-Naz Hayat Khan ${ }^{\mathrm{a}, \mathrm{d}}$, Andrew J. Ringer ${ }^{\mathrm{b}, \mathrm{c}}$
}

\begin{abstract}
Stroke is a second leading cause of death and disability in both developed and developing countries. $8-10 \%$ of ischemic strokes are caused by stenosis consequent to intracranial atherosclerotic disease. A growing body of evidence suggests that an aggressive approach may be indicated for patients with intracranial stenosis in the face of persistent symptoms despite adequate medical management, as the incidence of stroke or death in such situations is greater than $50 \%$. By comparison, patients who fail medical management and intravenous thrombolysis, the risk of neurological complications from angioplasty, stenting, or both together, has been reported as $0 \%$ to $28 \%$. The technical success rates for immediate, delayed and rescue angioplasty are very good (71-100\%). Stenting is an option following angioplasty of a stenosed vessel and in case of embolic strokes where angioplasty alone produces only a temporary recanalizing effect. The rate of stroke and death from intracranial angioplasty and stenting is up to $10 \%$, which is favorable when taking into consideration the risks of failed medical management. Currently, endovascular intervention is usually undertaken when medical management fails. Studies demonstrate angioplasty and stenting to be safe and effective. Technical success rate of angioplasty and stenting is up to $98-99 \%$. In this review, we address the indications for intracranial angioplasty and stenting and provide a reasonably detailed account for readers who perform these procedures. We will also address the potential complications that might arise during intervention and provide practical tips towards successful resolution.
\end{abstract}

Manuscript accepted for publication August 8, 2011

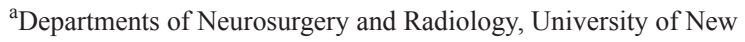
Mexico, Albuquerque, NM, USA

${ }^{\mathrm{b}}$ The University of Cincinnati (UC) Neuroscience Institute: Department of Neurosurgery, UC College of Medicine, Cincinnati, OH, USA

${ }^{\mathrm{c}}$ Mayfield Clinic, Cincinnati, OH, USA

${ }^{\mathrm{d}}$ Corresponding author: Shah-Naz Hayat Khan, Department of Neurosurgery, University Of New Mexico Health Sciences Center, MSC10 5615, 1 University of New Mexico, Albuquerque, NM 87131-0001, USA. Email: khan@sasktel.net; SHKhan@salud.unm.edu

doi:10.4021/jnr31w
Keywords: Stroke; Intracranial angioplasty; Intracranial stenting; Endovascular techniques

\section{Introduction}

Stroke is the second leading cause of death in the developed and third leading cause of death in the developing countries. It is the third leading cause of death ( 1 death every 3.5 minutes) and first leading cause of disability in North America $[1,2]$. Eight to ten percent of ischemic strokes are caused by stenosis consequent to intracranial atherosclerotic disease.

The current guidelines recommend intravenous (IV) thrombolysis if treatment is instituted within 3 hours of onset of anterior circulation stroke. The treatment window is longer for posterior circulation strokes, due to a lesser likelihood of hemorrhagic conversion of infarct [3]. Medical management for unstable, transient ischemic symptoms or crescendo transient ischemic attacks (TIA) may also include IV heparin or glycoprotein IIb/IIIa inhibitors such as abciximab or integrilin. If an isolated lesion such as cervical carotid stenosis is responsible, then urgent endarterectomy is indicated [4].

A growing body of evidence suggests that a similar aggressive approach may be indicated for patients with intracranial stenosis in the face of persistent symptoms despite adequate medical management, as the incidence of stroke or death in such situations is greater than 50\% [5]. In these patients who fail medical management and intravenous thrombolysis, the risk of neurological complications from angioplasty, stenting, or both together has been reported as $0 \%$ to $28 \%[6,7]$. Angioplasty alone is a viable option. The technical success rate for immediate (acute angioplasty performed for $>70 \%$ residual stenosis immediately after thrombolysis) and delayed angioplasty (performed for symptomatic restonosis or reocclusion within several hours or few days) is up to $100 \%$. For rescue angioplasty (performed when there is persistent vessel occlusion or stenosis because of ineffective chemical or mechanical thrombolysis), it is 71\% [8-10]. Stenting is a consideration following angioplasty of a stenosed vessel. It is also indicated in embolic strokes where angioplasty alone produces only a temporary recanalizing 
effect [11]. The rate of stroke and death from intracranial angioplasty and stenting is $9.5 \%$ [12]. The rate of restenosis is $32.4 \%$ to $35 \%$, of which $50 \%$ become symptomatic $[13,14]$. The option of conventional surgical intervention fell into disfavor following the EC/IC bypass study, which demonstrated no advantage for EC-IC bypass and greater rates of fatal and non-fatal strokes [15].

The above mentioned statistics favor endovascular intervention when medical management or IV recombinant tissue plasminogen activator (rt-PA; Activase ${ }^{\circledR}$; Genentech, San Francisco, CA) fail. Additionally, the rate of recanalization with IV rt-PA is up to $30 \%$ [16], necessitating the availability of bridging therapies. With intra-arterial TPA, the recanalization rate is $50-60 \%$ [11]. Studies demonstrate angioplasty and stenting to be safe and effective [9, 11, 17]. Technical success rate of angioplasty and stenting is up to $98-99 \%$ [11, $18,19]$.

\section{Procedural Overview}

\section{Indications}

Chemical thrombolysis in the form of intravenous rt-PA, is indicated when the stroke patients present within the three hour time frame and have an NIHSS score of less than 20 [20]. The endovascular treatment of stroke commonly include the options of intra-arterial chemical thrombolysis as well as, mechanical thrombolysis and thrombectomy. Intraarterial rt-PA is reserved for patients where angiography may be performed and treatment administered within 3 and 6 hours after symptom onset with an NIHSS score of greater than 4, or those with an NIHSS score of greater than 20 and the ability to treat within 6 hours $[3,21]$. Before treatment, CT scan is performed to rule out a hemorrhagic infarct or those with hypodensity or mass effect consistent with evolving infarct involving more than one-third of middle cerebral artery territory. A growing number of centers are also using $\mathrm{CT}$ perfusion or a similar study, to assess the feasibility of intervention. Such studies are anticipated to largely replace time as an intervention parameter. Thrombolytic devices such as Merci (Concentric Medical, Mountain View, CA) and Penumbra (Penumbra, Alameda, CA) systems are available as options for recanalization and have variable efficacy $[5,22,23]$. However, reopened arteries may reocclude consequent to thrombosis or vasospasm. In such cases, rescue angioplasty with or without stenting may prevent reocclusion in a stenotic artery and permit distal infusion of thrombolytic agents [9]. In addition, mechanical disruption of an occlusive thrombus using a mechanical retrieval device or balloon angioplasty may fragment the thrombus and present a greater surface area for thrombolytic agent binding and may improve the efficacy of chemical thrombolysis [14].

To this end, angioplasty is a consideration in symptom- atic intracranial stenosis that is focal and in an accessible location, namely, proximal to A2, M2 and P2. The addition of stenting becomes a consideration when vessel dissection occurs at site of stenosis, or it becomes apparent that the vessel is unlikely to remain patent with chemical thrombolysis and angioplasty alone.

\section{Preoperative management}

Maintain two intravenous lines, preferably $18 \mathrm{G}$ or larger. Insert a Foley catheter.

Verify the laboratory values including Platelet count, BUN, CR, APTT, PT/INR, and $\beta$-HCG for females in the reproductive age group. In case of renal insufficiency, diabetes, congestive heart failure and so on, ensure usage of diluted non-ionic contrast agent and carefully pre-plan to maintain contrast load to minimum. Additionally, patients' allergy to metals such as nickel and any unusual hypergoagulable state should be known. Both may preclude the use of stents.

In an acute situation where there is no opportunity to pre-medicate for endovascular intervention and stenting is anticipated, load the patient with Clopidogrel (Plavix ${ }^{\circledR}$; Bristol-Myers Squibb Co., Bridgewater, NJ and sanofi-aventis, Kansas city, MO) $600 \mathrm{mg}$ ( 8 tablets) orally prior to procedure, if patient is capable of safe oral intake. The loading dose may be reduced to $300 \mathrm{mg}$ ( 4 tablets) if it can be given at least 6 hours prior to intervention. Usually, clopidogrel is administered as $75 \mathrm{mg}$ PO daily starting 3 - 5 days before procedure. Alternatively, Abciximab (Reopro ${ }^{\circledR}$, Eli Lilly and Co., Indianapolis, IN) may be used in a loading dose of 0.25 $\mathrm{mg} / \mathrm{kg}$ followed by continuous IV infusion of $0.125 \mu \mathrm{g} / \mathrm{kg} /$ $\min (\max .10 \mu \mathrm{g} / \mathrm{min}$ ) for 12 hours. If abciximab is used, it should be administered after vascular access has been achieved and secured for intervention. If stenting is completed, continue administration of clopidogrel $75 \mathrm{mg}$ orally the same evening and then $75 \mathrm{mg}$ per os (PO) daily for at least 4 weeks. Additionally, the patient is placed on aspirin $81-325$ $\mathrm{mg}$ PO daily. In an acute stroke, time is of the essence and the preoperative preparation as well as the procedure should be performed with urgency, so as not to squander the narrow time frame available for intervention.

\section{Technique}

Intraoperative monitoring should include pulse oximetry, $\mathrm{ECG}, \mathrm{O}_{2}$ saturation, cardiac rate and rhythm, respiratory rate and blood pressure. Femoral artery access is secured with a 6 French sheath (e.g., Pinnacle; Terumo Interventional Systems, Tokyo). In older individuals or those with significant peripheral vascular disease, a longer sheath $(45 \mathrm{~cm})$ or a shuttle sheath $(90 \mathrm{~cm})$ may be necessary. If the use of Merci ${ }^{\circledR}$ or penumbra ${ }^{\circledR}$ device is planned, a larger caliber $(8$ or $9 \mathrm{Fr}$ ) sheath may be required. Consider placing one from the outset, or have one readily available to make a quick exchange 

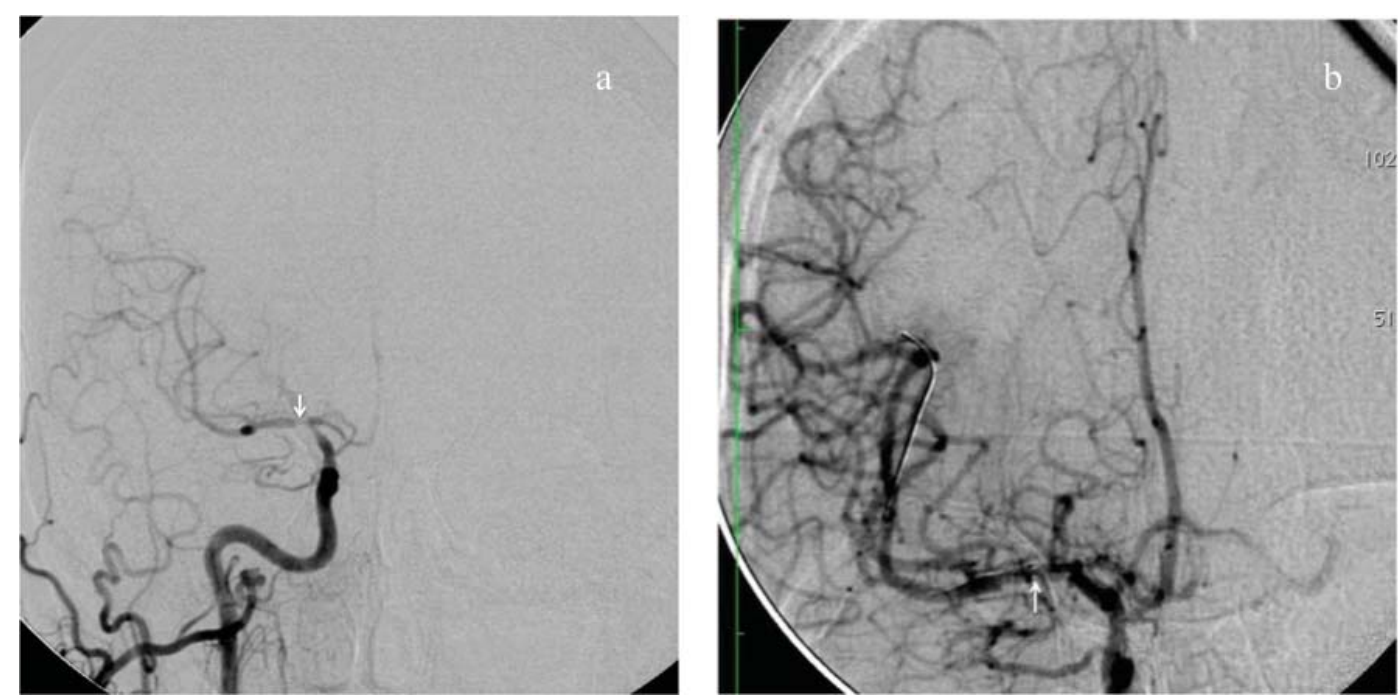

Figure 1. (a) Right common carotid artery (ICA) injection demonstrating symptomatic stenosis involving right internal carotid artery and particularly middle cerebral artery (MCA; arrow). (b) Angioplasty and stenting of ICA and MCA resulting in significant reconstitution of blood flow in the anterior and middle cerebral arteries. The tip of the guidewire is in the opercular segment of MCA, well distal to the area of stenosis. The arrow points to the distal radiopaque markers on the stent extending from ICA to MCA.

when the need arises.

If the location of cerebral lesion is uncertain, the site of occlusion and any collateral flow may be assessed quickly with a $5 \mathrm{Fr}$ pigtail catheter in the aortic arch. Center the image intensifier or detector over the patient's head, ensuring both right and left sides are in the field and inject contrast at a rate of $20 \mathrm{~mL} / \mathrm{sec}$ for a total of $30 \mathrm{~mL}$. This will permit identification of the site of occlusion as well as defining any collateral flow.

Once the lesion is defined and deemed suitable for angioplasty and or stenting, administer heparin intravenously to target an activated clotting time (ACT) of $300-350$ seconds in cases performed for TIAs. This can usually be achieved with a dose of $50-70 \mathrm{IU}$ of heparin per kg body weight. The $\mathrm{ACT}$ is tested hourly throughout the procedure and heparin administered IV to maintain the target ACT. In cases of acute stroke, a lower dose of 2000 - 3000 IU may be administered and supplemented by a continuous infusion of $400 \mathrm{IU} / \mathrm{h}$. Prepare a 6F guide catheter (Envoy; Cordis Endovascular Systems, Miami Lakes, FL) with continuous heparinized saline flush which is regulated with a pediatric transducer between the tubing, to ensure the heparinized saline flush runs continuously at a rate of $30 \mathrm{~mL} / \mathrm{hr}$.

Ensure that the carotid and vertebrobasilar vasculature have been satisfactorily visualized prior to catheterizing, in order to be cognizant of any asymptomatic pathology including stenosis or atherosclerotic plaque. It is also necessary to pay attention to the likelihood of tandem lesions.

Advance and position the guide catheter to provide support in the affected artery, remaining proximal to the lesion. When there is evidence of arterial occlusion in a distribution consistent with the acute neurological deficit, consider intraarterial thrombolysis. Thrombolysis is performed with an infusion microcatheter (Rapidtransit; Cordis Endovascular Systems, Miami Lakes, FL) guided to the site of the lesion via the guide catheter over a microguidewire (Transcend; Boston Scientific, Natick, MA), using road mapping and fluoroscopy. Whenever possible, advance the microcatheter distal to the site of occlusion and gradually pull back through the clot to permit infusion of thrombolytics distal to, within, and proximal to the occluded segment [9]. We use rt-PA and $1-2 \mathrm{mg}$ is administered manually distal to the major occlusion, followed by an infusion of $0.5 \mathrm{mg} / \mathrm{mL}$ at $20 \mathrm{~mL} / \mathrm{hr}(10$ $\mathrm{mg} / \mathrm{hr}$ ). The infusion is prepared by mixing $10 \mathrm{mg}$ of TPA in $20 \mathrm{~mL}$ on normal saline, resulting in a concentration of $1 \mathrm{mg}$ rt-PA per $2 \mathrm{~mL}$ saline (or $0.5 \mathrm{mg} / \mathrm{mL}$ ). Perform angiography every 15 minutes (following infusion of $2.5 \mathrm{mg} \mathrm{rt}-\mathrm{PA}$ ) as the catheter is gradually drawn back through the clot. Recross the lesion after each 15 minute angiogram if the artery is still occluded, inject 1 - 2 mg rt-PA manually and resume the t-PA infusion. The maximum dose administered intra-arterially (including boluses and infusion) is $22 \mathrm{mg}$. The calculation of maximal intra-arterial dose does not include the intravenous infusion, which is $0.6 \mathrm{mg} / \mathrm{kg}$ according to our protocol [21]. Infusion is discontinued when 1) adequate recanalization is achieved, 2) extravasation of contrast material is noted on angiography, 3) a maximum dose of $50 \mathrm{mg}$ has been administered, or 4) the administered dose approaches the maximum dose without clinical or angiographic improvement. Other options for intra-arterial thrombolysis include abciximab, urokinase and prourokinase. If thrombolysis results in inadequate or failed recanalization, angioplasty may be 


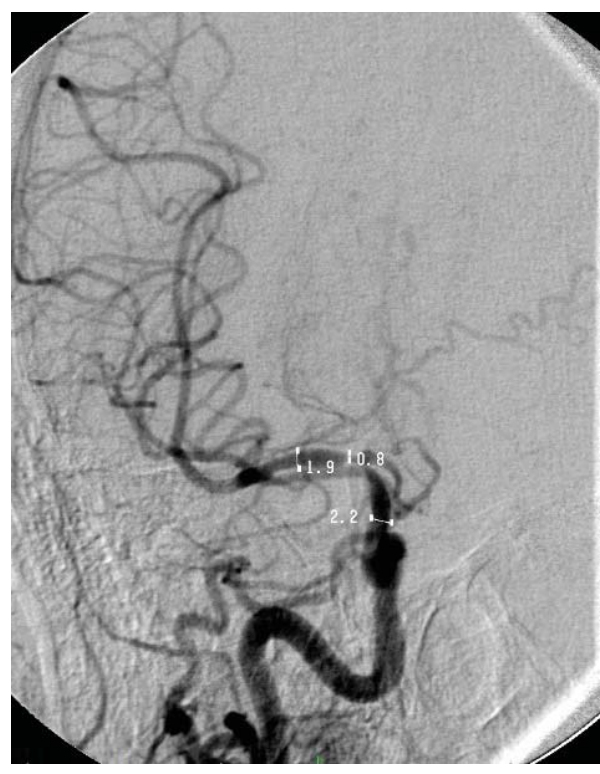

Figure 2. Measurements of width of stenosed segment of MCA for stenting. The vessels are measured proximal (ICA) and distal (MCA) to the lesion to enable appropriate stent size selection. The length of the stent is at least 2 $\mathrm{mm}$ longer at either end of the stenosed segment.

performed (see 'Angioplasty' below) [9].

In case stenosis is the cause of presenting symptoms, angioplasty and stenting may be indicated (Fig. 1). Measure the length and width of the stenotic lesion along with, the width of normal vessel proximal and distal to the stenosed segment, on angiography. Select an angioplasty balloon which is approximately $75-80 \%$ the size of the artery proximal and distal to the stenosis (Fig. 2).

If stenting is needed, select a stent with a diameter equal to or slightly greater than the normal caliber of the vessel. The length of the stent should be at least $2-3 \mathrm{~mm}$ beyond the proximal and distal aspect of stenosis each. Prior to commencing angioplasty, again ensure the guide catheter is appropriately positioned to support the angioplasty microcatheter and stent systems. A working view is selected that elongates the target artery with adequate magnification. In addition to the roadmap, a native live fluoroscopy image should be available at all times.

\section{Angioplasty}

The goal of angioplasty should be to dilate the stenosis to approximately $75-80 \%$ of the normal vessel proximal and distal to stenosis. Attempting to achieve normal diameter or overdilating the artery may result in dissection or vessel rupture. If the angioplasty is being performed for embolic occlusion, the goal is reestablishment of blood flow through the occluded vasculature.

For the purpose of preparing the balloon catheter, pre- pare a dilute solution of approximately $2 / 3$ contrast and $1 / 3$ saline for balloon inflation. It is our practice not to purge the balloon catheter outside the patient, so that it retains its original smooth contour while crossing the lesion. Instead, we perform a negative pressure preparation after the balloon is positioned across the lesion by aspirating the inflation lumen with a large $(20 \mathrm{~mL})$ syringe containing $2-3 \mathrm{~mL}$ of the dilute contrast solution and then releasing the negative pressure created. If the syringe is held upright, the air from the inflation lumen will rise as bubbles in the syringe and then be refilled with the contrast solution.

\section{Preparation of inflation device with manometer}

Fill a $20 \mathrm{~mL}$ syringe with $10 \mathrm{~mL}$ contrast and $5 \mathrm{~mL}$ heparinized saline (2/3:1/3 concentration). Attach a Three-way stopcock to the inflation device and connect the $20 \mathrm{~mL}$ syringe containing contrast medium to the sideport of the stopcock. Aspirate the contrast into the inflation device, leaving only $2-3 \mathrm{~mL}$ within the syringe. Purge any air out of the barrel and tubing of the inflation device, all the way to the distal portal of the stopcock. Leave inflation device on table, until it is needed.

\section{Deployment of PTA balloon catheter for angioplasty}

Ensure the dual-lumen, or "over the wire", balloon catheter (Gateway' ${ }^{\mathrm{TM}}$; Boston Scientific, Fremont, CA) is continuously flushed with heparinized saline as it is loaded with a 0.014 " exchange length $(300 \mathrm{~cm})$ microwire and inserted into the guide catheter. Once the distal aspect of balloon catheter is within the lumen of guide catheter, advance the microwire so that it leads the balloon catheter. Continue to advance the balloon catheter and wire assembly until the proximal markings on the balloon catheter align with the hub of the guide catheter RHV, indicating that the balloon catheter tip has reached the distal tip of the guide catheter. Perform fluoroscopy to confirm location of the microcatheter, wire and guide catheter. Perform angiography in the appropriate working views, by hand injection of contrast using the free port of the stopcock connected in the guide catheter system, to visualize and treat the lesion. Using road map guidance, advance the wire with constant half rotatory motion to cross the lesion. Maintain adequate length of wire distal to the lesion to support the balloon catheter during advancement, until the two marker bands on the balloon catheter tip which indicate the proximal and distal ends of the angioplasty balloon are positioned across the lesion. For better visualization of markers also use concurrent native imaging and magnification, as necessary. Perform angiography to ascertain appropriate positioning of the balloon relative to the stenosis. Repeat angiography every time the position of the balloons is adjusted, until completely satisfied with the position of the balloon.

Using the inflation device filled with dilute contrast, 
aspirate the syringe (with nozzle pointing down) attached to the sideport of the stopcock to purge air out of the balloon angioplasty system. Slowly release the plunger of the syringe, so that the air is replaced with the contrast left in syringe for purpose. Repeat this step at least 1 - 2 times to ensure all air has been purged from the balloon catheter. Turn the stopcock, so that it becomes open to the inflation device. Using the chart provided with the balloon catheter to predict the balloon diameter during inflation, slowly begin inflating the angioplasty balloon. Inflate slowly by turning the screw provided on the inflation device at a rate $\leq 1 \mathrm{~atm} / 15 \mathrm{sec}$ (to "stretch" not "crack" the vessel). Perform fluoroscopy frequently to visualize progression of angioplasty. During inflation, the balloon may acquire a "waist" due to the stenosis. This resolves with the progression of angioplasty. Once the goal pressure is reached, oscillate from 1 to 2 atm above that pressure for 10 to 30 seconds before deflation. Results of angioplasty and complete deflation of balloon are evaluated immediately by angiography. In order to achieve complete deflation, the stopcock may need to be opened to the syringe to aspirate the catheter. Close the stopcock to syringe while aspiration is fully applied and confirm complete balloon deflation fluoroscopically. If needed, angioplasty may be repeated by following the steps described above.

\section{Removal of PTA balloon catheter}

Confirm complete balloon deflation by performing fluoroscopy. Under continuous fluoroscopy, begin catheter withdrawal while the guidewire remains in position across the lesion. This is a two-person maneuver: The primary operator controls the guide catheter at the hub of the RHV and the second operator withdraws the balloon catheter over the wire while constantly observing the tip of the wire on the monitor to ensure it is not withdrawn inadvertently. The entire assembly is kept straight to ensure adequate control and maneuverability. The second operator progressively steps away from the first while withdrawing the catheter over the wire to keep the system straight. Be vigilant to ensure the wire and catheter are not contaminated during the process by contact with unsterile surfaces. As soon as the distal tip of the balloon catheter exits the hub of RHV, the first operator grabs the wire and secures it by forming a small loop with it and holding it just next to the hub. The RHV knob is tightened around the wire. Then the first operator uses a moist Telfa TM strip to wipe off the extraneous wire proximal to distally as the second operator completes the removal of the catheter off the wire.

Wipe off the balloon catheter with heparinized saline and store in a bowl with the same solution, in case it may be required for reuse. Perform angiography to assess and document results. If satisfied the wire may be withdrawn. If stenting is planned as the next step, maintain wire access access across lesion.

\section{Stenting}

Select a stent of the same size as or slightly larger than the normal size of the vessel to be stented. An undersized stent risks forward migration and/or dislodgement. The stent should extend at least $2 \mathrm{~mm}$ beyond the stenosed segment at either end.

Below follows the technique of stent deployment using the Wingspan ${ }^{\mathrm{TM}}$ (Boston Scientific Fremont, CA) stent system.

\section{Selection of appropriate stent}

Study the angiogram to select the appropriate size of stent. Measure the normal vessel diameter proximal and distal to the lesion. Select a stent equivalent to or slightly larger than the diameter of the normal vessel. The length of the stent should be such that at least $2 \mathrm{~mm}$ of the stent extends proximal and distal to the lesion, for example, for a vessel with a diameter of $4.4 \mathrm{~mm}$ and a lesion length of $4 \mathrm{~mm}$, use a 4.5 $\mathrm{mm}$ stent (which expands to $4.9 \mathrm{~mm}$ ) with a $9 \mathrm{~mm}$ or longer length (refer to table provided on package insert of product for sizing guidelines)

\section{Preparation of stent}

Using a $15 \mathrm{~mL}$ syringe, flush the dispenser hoop with saline. Carefully pull out the proximal hub aspect of the system from the tray. Locate the RHV of the outer catheter and tighten it onto the inner stabilizing catheter of the stent system. Transfer the entire system out onto the table from the tray and dispenser hoop. Inspect the stent system for damage or flaws including the position of the stent near the distal tip of the catheter. Attach an RHV to the hub of the inner catheter, which is the most proximal aspect of the system. Flush the lumen of the inner catheter with heparinized saline. Loosen the previously tightened RHV of the outer catheter and flush the lumen with heparinized saline to purge any air. Gently advance the pusher of the inner catheter of the system until the proximal radiopaque markerband bumper is just proximal to the stent. Retighten the RHV of outer catheter to lock onto the inner catheter, so that the entire system will move as a single unit. There is an option of connecting heparinized saline flush to the side ports of the RHVs of the outer and inner catheters.

\section{Deployment of stent}

Fluroscopically ascertain that the guidewire is still across the lesion. Perform angiography in working view. Backload the stent system onto the guidewire. Loosen the hub of the RHV of the guide catheter and advance the stent system into it. When the stent system is just within the distal tip of the guide catheter, perform a roadmap to assist in navigation. Advance 
the stent system over the guidewire until the stent crosses the lesion and the distal marker bands on the stent are just distal to the lesion. Ascertain the positions of the distal tip of the guidewire, distal marker bands on the distal tip of the stent, proximal marker band on the stent pusher/bumper. The distal tip of the guidewire should be crossing the lesion and well distal to the distal marker bands on the stent. The distal marker bands on the stent are kept just distal to the distal aspect of the lesion. The proximal marker bands are proximal to the lesion. Typically we achieve this by first advancing the stent markers distal to the lesion, then pulling back into position across the lesion. This ensures that redundancy has been removed from the delivery catheter to limit forward migration of the stent during delivery. Perform angiography and if needed, adjust the position of stent. Repeat angiography after each adjustment.

Loosen the RHV valve of the outer stent delivery catheter and slightly withdraw the hub of the outer catheter until the stent is directly aligned with the lesion. Tighten the RHV and if needed pull back on the system to ensure that all slack has been removed and the system is completely straight. Perform angiography to ascertain satisfactory final position. Ensure that vessel distal to the lesion is visualized. Loosen the RHV of the outer catheter.

Now deploy stent under continuous fluoroscopy by stabilizing the inner catheter with one hand while withdrawing the hub of the outer catheter with the other hand. Watch on fluoroscopy as the markerbands at the distal end of the stent spread out into multiple smaller markers, indicating the opening of the stent. Continue deployment in a single smooth motion until the stent is completely deployed. Do not attempt to move the stent or advance the outer catheter once the deployment is underway. After completion of deployment, retighten the RHV of the outer catheter and gently withdraw the entire system maintaining wire access across the lesion. Once the system is out of the guide catheter RHV, secure the wire by forming a loop and holding it right at the guide catheter hub. Stop fluoroscopy and remove the system off the wire. This is a two person maneuver performed as described above for removal of the angioplasty balloon.

Perform angiography including, standard AP and lateral views and before relinquishing wire access. Ascertain satisfactory result by comparing it to pre-procedure angiogram to rule out any distal vessel cut off or change indicative of embolism. After successful angioplasty and stenting, revascularization of the previously occluded or stenotic vessels will be apparent. If the procedure was performed under conscious sedation, as is our preference, examine the patient carefully for any new neurological deficits. Withdraw the guide wire. If percutaneous closure of the groin access site is intended, retract the guide catheter to the femoral artery and perform femoral angiogram in $\approx 45^{\circ}$ ipsilateral oblique view. If the arteriotomy is proximal to femoral bifurcation and the vessel size is $\geq 4.9 \mathrm{~mm}$, we prefer to perform closure with Angi- oseal (St. Jude Medical, St. Paul, MN). Cover the access site with a sterile dressing.

\section{Alternative stent system}

We are increasingly using Enterprise ${ }^{\mathrm{TM}}$ Vascular Reconstruction device (Cordis Neurovascular, Miami Lakes, FL) in vessels $<4.5 \mathrm{~mm}$ because of its ease of deployment, which is superior to other systems currently in market. Its limitation is the availability in only a single diameter: $4.5 \mathrm{~mm}$. A brief description of its deployment is as follows:

Advance and navigate a 0.021 Prowler Select Plus (Cordis) catheter attached to continuous flushing solution of heparinized saline, over a 0.010 or 0.014 microguidewire. The tip of catheter is placed distal to the stenosis. Carefully remove the vascular reconstruction device from its package. Release the delivery wire from the clip on the dispenser hoop and grasp the delivery wire and introducer together when removing the device system from the hoop, to prevent stent movement. Ensure the entire system is damage free and that the tip of the delivery wire is within the introducer. Insert the distal end of the introducer partially into the RHV on the Prowler microcatheter. Tighten the RHV around the introducer and flush the RHV to remove any air bubbles and confirm that the saline exits the proximal end of introducer. Advance the introducer until it fully engages with the microcatheter and tighten the RHV. Advance the delivery wire to transfer the stent from the introducer into the microcatheter. Continue to advance the wire into the microcatheter until the delivery wire reference marker $(150 \mathrm{~cm}$ from the wire tip) enters the introducer. Loosen the RHV and remove the introducer. From this point onwards, use fluoroscopy to track the stent to the tip of microcatheter. Position the stent such that it extends $5 \mathrm{~mm}$ beyond the stenotic lesion both proximally and distally, by aligning the stent positioning marker on the delivery wire with the lesion. After the stent is appropriately positioned, carefully retract the microcatheter while maintaining the delivery while stationary in position. The stent expands as the microcatheter is retracted. If positioning is unsatisfactory, the stent can be recaptured and repositioned, until the point where the proximal end of the stent positioning marker is aligned with the distal markerband on the microcatheter. Maintain some tension on the delivery wire to keep the stent stable. Do not attempt to recapture and reposition more than once. Once the stent is completely detached, advance and position the microcatheter distal to the stent. In this manner, access will still be maintained after removal of delivery wire. Remove and discard the delivery system.

\section{Potential complications and complication management}

Problems encountered during angioplasty and solutions

1) Problem: Tandem lesions. 
Determine if there is any benefit in dilating the proximal lesion, if distal lesion is inaccessible. Determine if both lesions need dilatation and if the same can be performed.

2) Problem: Inability to access the lesion or maintain access because of tortuous vasculature.

Maneuver: Place a longer sheath (e.g., $45 \mathrm{~cm}$ ) or a Shuttle sheath $(90 \mathrm{~cm})$ (Cook, Indianapolis, IN) for better support. In case of anterior circulation lesion, if need be, after placing the Shuttle sheath in the superior aspect of descending aorta, advance an $\mathrm{H} 1$ catheter over guidewire into the common carotid artery (CCA) of interest. Then advance the Shuttle sheath over the guide wire and $\mathrm{H} 1$ catheter into the CCA.

Consider percutaneous access other than femoral, for example, radial or brachial. Direct carotid access also largely eliminates tortuous vasculature. However, the risk of complications including potentially fatal emboli, increases.

3) Problem: Balloon catheter will not move forward despite advancing it.

This may be consequent to the guide catheter losing its position and dropping back into the external carotid artery (ECA), CCA, or further proximally.

Maneuver: Locate the tip of the guide catheter. In case it has dropped down, loosen the hub of the RHV attached to the guide catheter. Remove the redundancy in the system by pulling back the balloon catheter and guidewire within it concurrently, until the loops are gone and the system is straightened out. Advance the guide catheter over the microcatheter and microwire back into intended position.

4) Problem: Unable to cross the lesion with the balloon catheter

Maneuver: Use a smaller size balloon catheter to cross the lesion. Perform angioplasty to "pre-dilate" the lesion. Then, re-attempt deployment of balloon catheter of intended size alternatively, use a sequential dilatation technique. With the uninflated balloon positioned in the proximal end of the stenosis, inflate the balloon. Then deflate, advance the balloon further, re-inflate and repeat as necessary until the entire lesion can be crossed ("bump and run" technique).

5) Problem: Disconnection of inflation device during angioplasty.

Maneuver: attach large barrel syringe to Balloon (side) port of catheter and aspirate to completely deflate balloon prior to withdrawal.

6) Problem: Lack of flow distal to angioplasty on followup angiography. This is probably due to acute vasospasm or dissection caused by the angioplasty.

Maneuver: Maintain wire access across the lesion. Rapidly deploy the stent across the lesion. Consider repeating the angioplasty. Also consider administration of verapamil through the microcatheter if vasospasm is suspected, following completion of angioplasty and stent deployment.

7) Problem: Proximal portion/tip of guide wire contami- nated by touching unsterile surface, for example, the injector or IV poles during angioplasty. The maintenance of wire access across the lesion is imperative. Therefore, the wire must not be withdrawn and discarded.

Maneuver: Use an alcohol swab to wipe off the exposed wire. As extra precaution, repeat wipe with a second swab.

\section{Problems encountered during stenting and solutions}

1) Problem: Difference in size of vessel proximal and distal to stent.

Maneuver: Choose a self-expanding stent using the larger size measured, for example, if the basilar artery measures $4 \mathrm{~mm}$ and the posterior cerebral artery is $2 \mathrm{~mm}$. The size of the stent should be $\geq 4 \mathrm{~mm}$.

2) Problem: Resistance in advancing the stent system.

Maneuver: Ensure the system is straight and there is no redundancy which may be causing excessive tension build up in the system. Slightly retract the stent system and guidewire to remove tension. Check that the guide catheter is positioned adequately to support the system. If not, advance it forward. If there are any loops in the stent system, straighten them out. For wingspan stent, consider switching to a soft (floppy) guidewire which enables easy maneuverability of the stent system rather than, the more firm support guidewires. Ensure that the flush system is functioning. Alternatively, if a soft wire is being used, then consider changing it for a stiffer wire, which may provide better support.

Once the stent system is advancing, continue to advance it even if it goes distal to the lesion because, it is easier to move the system from distal to proximal location than vice versa. If inordinate resistance continues despite corrective maneuvers, consider the possibility of damage to the guidewire from use and discard it (in case of wingspan ${ }^{\mathrm{TM}}$ system) for a new wire. At the same time, examine and consider replacing the stent system with a new one as well.

3) Problem: Feeling of resistance while deploying stent.

Maneuver: Some resistance is usually encountered and expected. However, if the resistance is excessive, check to ensure the entire system is straight and there is no tension buildup. Slightly retract the system and guidewire to remove excessive tension.

Check the position of guide catheter to ensure it is providing adequate support. If not, advance it further up. If excessive tension persists, consider discarding the stent system and replacing it with a new system. If deployment is already underway, do not attempt to resheath the stent. Complete the deployment. However, the Enterprise ${ }^{\mathrm{TM}}$ system, can be resheathed once during deployment, provided this is performed within the recapturability Limit, which is up to the point where the proximal end of the stent positioning marker is aligned with the distal markerband on the microcatheter.

4) Problem: Loss of position during deployment.

Maneuver: If the Wingspan stent has migrated distally, 
it may be possible to carefully pull back the entire system as deployment is taking place until it is back in the desirable location. Then continue with the deployment. If any difficulty is encountered in the retraction, just deploy the stent in its current location. Then, deploy a second stent to cover the desired location.

In case the stent has migrated proximal to the lesion while deployment is underway, do not attempt to push it forward to its intended location. Just deploy the Wingspan stent maintaining wire access and then use an additional stent to cover the more distal site. The Enterprise Stent may be resheathed once and then repositioned.

5) Problem: Only partial coverage of lesion by the stent.

Maneuver: Maintain access across the lesion. Discard the used stent system. Bring a new system into the field, which should be greater than or equal to the size of the first system. Deploy the second stent within the first (telescoping) so that the entire lesion is covered including, at least $2 \mathrm{~mm}$ of normal vessel on either side.

6) Problem: Resistance during retraction of system following stent deployment.

Maneuver: Ensure the entire system (inside and outside the patient) is straight and there is no tension buildup. Slightly retract the system and guidewire to remove excessive tension. Maintain guidewire access at all times across the lesion. If tension persists, Loosen the RHV of the outer body of the stent system and pull back the hub of the inner body, until its tip is in contact with the tip of the outer body. Retighten the RHV of the outer body and remove the entire stent system.

7) Problem: Wire is entangled within the stent and cannot be retracted.

Maneuver: This most likely occurs during wire loading prior to delivering the stent. In this situation, it is important to stabilize the stent during wire withdrawal to prevent migration. Advance an angioplasty balloon over the wire until it meets resistance at the point where the wire crosses the stent struts. Inflate the balloon to nearly match the artery diameter, stabilize the balloon catheter, then forcibly withdraw the wire.

8) Problem: Breakage/mechanical failure of stent during deployment.

Maneuver: If the stent is only partially deployed and still partially sheathed consider retracting the entire system along with the stent outside the patient or, if complete retraction is not possible, retracting to deploy the stent in a more neutral location, for example, ECA or its branches.

In case the stent is completely outside the sheath, retract and discard the stent system. Introduce a microcatheter (e.g. Excelsior SL 10 or Prowler Select Plus) over the wire. Use the microcatheter to introduce an alligator snare or a similar retrieval device. Grab the stent and pull to the tip of the catheter. If possible, withdraw the stent and microcatheter into the guide catheter. Once within the guide catheter, remove the entire system, except the wire. If the stent cannot be retracted into the guide catheter, maintain it at the tip of the catheter and pull out the entire system, except the wire.

\section{Conclusion}

Stroke is a common emergency, which will become more pervasive as the population continues to age. Time is of the essence in successful management of stroke. The current recommendations are to perform endovascular intervention if within the three to 6 hours window for anterior circulation strokes. In case of posterior circulation strokes, the time limitations are less clear and intervention may be attempted within 24 - 48 hours. Once vascular access is obtained, heparinize the patient with a goal of maintaining ACT from 300 to 350 through the case, in order to decrease the risk of iatrogenic embolic events. Alternatively, administer i.v. heparin 400 i.u./hr, after a 2000 - 3000 i.u. bolus. In case of technical difficulties due to tortuous vasculature address the problem expeditiously, rather than repeatedly trying the same maneuver to no avail. If a longer sheath does not solve the problem, consider alternative route of access to avoid some of the tortuosity, for example, consider the radial or brachial arteries. Maintain the guide catheter as close as safely possible and proximal to the lesion, to provide adequate support enabling successful deployment of balloon and stent. The entire system must be kept straight in order to avoid maneuvering difficulties or prolapse of the balloon or stent system. Overdilation during angioplasty or, undersizing of the stent must be avoided. In case a stent is placed, use intravenous heparin for atleast 12 hours and plavix for 4 weeks, until stent endothelialization has occurred, in order to avoid in-stent thrombosis.

\section{References}

1. Lopez AD, Murray CC. The global burden of disease, 1990-2020. Nat Med. 1998;4(11):1241-1243.

2. Helgason CM, Wolf PA. American Heart Association Prevention Conference IV: prevention and rehabilitation of stroke: executive summary. Circulation. 1997;96(2):701-707.

3. Adams HP, Jr., del Zoppo G, Alberts MJ, Bhatt DL, Brass L, Furlan A, Grubb RL, et al. Guidelines for the early management of adults with ischemic stroke: a guideline from the American Heart Association/American Stroke Association Stroke Council, Clinical Cardiology Council, Cardiovascular Radiology and Intervention Council, and the Atherosclerotic Peripheral Vascular Disease and Quality of Care Outcomes in Research Interdisciplinary Working Groups: the American Academy of Neurology affirms the value of this guideline as an educational tool for neurologists. Stroke. 2007;38(5):1655-1711. 
4. Rothwell PM, Eliasziw M, Gutnikov SA, Warlow CP, Barnett HJ. Endarterectomy for symptomatic carotid stenosis in relation to clinical subgroups and timing of surgery. Lancet. 2004;363(9413):915-924.

5. Thijs VN, Albers GW. Symptomatic intracranial atherosclerosis: outcome of patients who fail antithrombotic therapy. Neurology. 2000;55(4):490-497.

6. Boulos AS, Agner C, Deshaies EM. Preliminary evidence supporting the safety of drug-eluting stents in neurovascular disease. Neurol Res. 2005;27 Suppl 1:S95-102.

7. Gupta R, Schumacher HC, Mangla S, Meyers PM, Duong H, Khandji AG, Marshall RS, et al. Urgent endovascular revascularization for symptomatic intracranial atherosclerotic stenosis. Neurology. 2003;61(12):17291735.

8. Ueda T, Sakaki S, Nochide I, Kumon Y, Kohno K, Ohta S. Angioplasty after intra-arterial thrombolysis for acute occlusion of intracranial arteries. Stroke. 1998;29(12):2568-2574.

9. Ringer AJ, Qureshi AI, Fessler RD, Guterman LR, Hopkins LN. Angioplasty of intracranial occlusion resistant to thrombolysis in acute ischemic stroke. Neurosurgery. 2001;48(6):1282-1288; discussion 1288-1290.

10. Michels KB, Yusuf S. Does PTCA in acute myocardial infarction affect mortality and reinfarction rates? A quantitative overview (meta-analysis) of the randomized clinical trials. Circulation. 1995;91(2):476-485.

11. Levy EI, Ecker RD, Horowitz MB, Gupta R, Hanel RA, Sauvageau E, Jovin TG, et al. Stent-assisted intracranial recanalization for acute stroke: early results. Neurosurgery. 2006;58(3):458-463; discussion 458-463.

12. Cruz-Flores S, Diamond AL. Angioplasty for intracranial artery stenosis. Cochrane Database Syst Rev. 2006;3:CD004133.

13. Stenting of Symptomatic Atherosclerotic Lesions in the Vertebral or Intracranial Arteries (SSYLVIA): study results. Stroke. 2004;35(6):1388-1392.

14. Puetz V, Gahn G, Becker U, Mucha D, Mueller A, Weir NU, Wiedemann B, et al. Endovascular therapy of symptomatic intracranial stenosis in patients with impaired regional cerebral blood flow or failure of medical therapy. AJNR Am J Neuroradiol.
2008;29(2):273-280.

15. Failure of extracranial-intracranial arterial bypass to reduce the risk of ischemic stroke. Results of an international randomized trial. The EC/IC Bypass Study Group. N Engl J Med. 1985;313(19):1191-1200.

16. Wolpert SM, Bruckmann H, Greenlee R, Wechsler L, Pessin MS, del Zoppo GJ. Neuroradiologic evaluation of patients with acute stroke treated with recombinant tissue plasminogen activator. The rt-PA Acute Stroke Study Group. AJNR Am J Neuroradiol. 1993;14(1):313.

17. Levy EI, Siddiqui AH, Crumlish A, Snyder KV, Hauck EF, Fiorella DJ, Hopkins LN, et al. First Food and Drug Administration-approved prospective trial of primary intracranial stenting for acute stroke: SARIS (stent-assisted recanalization in acute ischemic stroke). Stroke. 2009;40(11):3552-3556.

18. Roubin GS, Yadav S, Iyer SS, Vitek J. Carotid stentsupported angioplasty: a neurovascular intervention to prevent stroke. Am J Cardiol. 1996;78(3A):8-12.

19. Fiorella D, Levy EI, Turk AS, Albuquerque FC, Niemann DB, Aagaard-Kienitz B, Hanel RA, et al. US multicenter experience with the wingspan stent system for the treatment of intracranial atheromatous disease: periprocedural results. Stroke. 2007;38(3):881-887.

20. Tissue plasminogen activator for acute ischemic stroke. The National Institute of Neurological Disorders and Stroke rt-PA Stroke Study Group. N Engl J Med. 1995;333(24):1581-1587.

21. Khatri P, Hill MD, Palesch YY, Spilker J, Jauch EC, Carrozzella JA, Demchuk AM, et al. Methodology of the Interventional Management of Stroke III Trial. Int J Stroke. 2008;3(2):130-137.

22. Smith WS, Sung G, Starkman S, Saver JL, Kidwell CS, Gobin YP, Lutsep HL, et al. Safety and efficacy of mechanical embolectomy in acute ischemic stroke: results of the MERCI trial. Stroke. 2005;36(7):1432-1438.

23. McDougall C, Clark W, Mayer T, doerfler A, Grunwald I, Berkefeld J, et al. The Penumbra stroke trial: safety and effectiveness of a new generation of mechanical devices for clot removal in acute ischemic stroke. International Stroke Conference; 2008; New Orleans, LA: American Stroke Association; 2008. 